\title{
Detection of Noble Gas Scintillation Light with Large Area Avalanche Photodiodes (LAAPDs)
}

\author{
R. Chandrasekharan M. Messina A. Rubbia \\ Institut für Teilchenphysik, ETHZ, \\ CH-8093 Zürich, Switzerland
}

\begin{abstract}
Large Area Avalanche Photodiodes (LAAPDs) were used for a series of systematic measurements of the scintillation light in $\mathrm{Ar}, \mathrm{Kr}$, and $\mathrm{Xe}$ gas. Absolute quantum efficiencies are derived. Values for $\mathrm{Xe}$ and $\mathrm{Kr}$ are consistent with those given by the manufacturer. For the first time we show that argon scintillation $(128 \mathrm{~nm})$ can be detected at a quantum efficiency above $40 \%$. Low-pressure argon gas is shown to emit significant amounts of non-UV radiation. The average energy expenditure for the creation of non-UV photons in argon gas at this pressure is measured to be below $378 \mathrm{eV}$.
\end{abstract}

Key words: Avalanche Photodiode, LAAPD, DUV detection, quantum efficiency, noble gas scintillation, argon IR emission

PACS:

\section{Introduction}

Noble gases are known to provide scintillation light with high yield, comparable to that of $\mathrm{NaI}(\mathrm{Tl})$. Detection of this light proves fundamental in many applications in which noble gas is used as medium (for example in TPCs the prompt scintillation light is used for triggering and $T_{0}$ determination). Difficulties arise from the fact that noble gas emission is peaked in the deep ultraviolet (DUV) range. To detect such light, wavelength shifter-coated Photomultiplier Tubes (PMTs) have often been used, resulting in a very low global quantum efficiency. There is interest in knowing if large area avalanche photodiodes (LAAPDs) could be a viable alternative, in particular in applications where the radiopurity of the PMT's glass is a concern (e.g. direct dark matter searches, detection of solar neutrinos, etc.). 
The results presented in this paper show that it is in principle possible to detect gas scintillation from $\mathrm{Kr}$, Xe and Ar with APDs with a quantum efficiency higher than with PMTs. The issues related to the signal to noise of APDs, to the parallel operation of a large number of APDs to increase the sensitive area and the mechanical problems at potential cryogenic temperatures remain to be solved.

\section{Noble Gas Scintillation}

Gas excitation and ionization can lead to the emission of scintillation photons in the DUV range via the processes [1] of excitation

$$
\begin{aligned}
R^{*}+R & \rightarrow R_{2}^{*} \\
R_{2}^{*} & \rightarrow 2 R+h \nu,
\end{aligned}
$$

and ionization

$$
\begin{aligned}
e_{\text {hot }}+(\text { collisions }) & \rightarrow e_{t h} \\
R^{+}+R & \rightarrow R_{2}^{+} \\
R_{2}^{+}+e_{t h} & \rightarrow R^{* *}+R \\
R^{* *} & \rightarrow R^{*}+\text { heat } \\
R^{*}+R & \rightarrow R_{2}^{*} \\
R_{2}^{*} & \rightarrow 2 R+h \nu .
\end{aligned}
$$

The possible emission of non-UV light, mostly in the IR region, is attributed to the transitions between excited atomic states. Indeed, the atomic spectrum of argon shows several very intense lines at wavelength 700-1050 nm. A set of lines located between 400-500nm has intensities below $3 \%$ of the most intense lines found in the infrared region.

According to $[3,4,5,6]$, the average energy expended per ion pair $W_{g}^{\text {ion }}$ can be related to the ionization potential $I$

$$
\frac{W_{g}^{i o n}}{I}=\frac{E_{i}}{I}+\left(\frac{E_{e x}}{I}\right)\left(\frac{N_{e x}}{N_{i}}\right)+\frac{\epsilon}{I},
$$

where $N_{i}$ is the number of ions produced at an average energy expenditure of $E_{i}, N_{e x}$ is the number of excited atoms produced at an average expenditure of $E_{e x}$, and $\epsilon$ is the average kinetic energy of sub-excitation electrons. Equation 3 can also be applied to condensed noble gas if $I$ is substituted by the band-gap energy $E_{g}$. Values used for calculations in this work are shown in Table 1.

The energy balance equation is energy dependent in all four every terms, however, for $E \gg I$ this dependence is weak. For $\alpha$-particles in argon, $W_{g}^{i o n}=$ 
$26.5 \pm 0.5 \mathrm{eV}$ at energies $E_{\alpha} \geq 1 \mathrm{MeV}$. For $E_{\alpha}=0.1 \mathrm{MeV}$, the value is only somewhat higher at $W_{g}^{\text {ion }}=27.5 \pm 1.0$, increasing further as the kinetic energy is reduced. Our measurements were performed in a pressure range where the scintillation is brought forth by $\alpha$ particles with at least $0.5 \mathrm{MeV}$ kinetic energy.

\begin{tabular}{|c|c|c|c|c|c|c|c|}
\hline & $W_{g}^{\text {ion }}$ & $N_{e x} / N_{i}$ & $\mathrm{I}$ & $\epsilon / I$ & $E_{i} / I$ & $E_{e x} / I$ & UV Peak Wavelength \\
\hline $\mathrm{Ar}$ & 26.34 & 0.4 & 15.7 & 0.33 & 1.06 & 0.85 & $128 \mathrm{~nm}$ \\
$\mathrm{Kr}$ & 24.1 & 0.4 & 13.9 & 0.36 & 1.06 & 0.85 & $150 \mathrm{~nm}$ \\
$\mathrm{Xe}$ & 21.9 & 0.4 & 12.1 & 0.39 & 1.1 & 0.85 & $175 \mathrm{~nm}$ \\
\hline
\end{tabular}

Table 1

Values used for calculations in this work. Energies are expressed in eV. From $[3,4,5,6]$.

Assuming no ionization contribution to UV scintillation light, justified at the low pressures used in this work $[2,7]$, the average energy expenditure per photon is

$$
W_{\gamma}^{D U V}=\left(\frac{N_{i}}{N_{e x}}\right) \cdot E_{i}+E_{e x}+\left(\frac{N_{i}}{N_{e x}}\right) \cdot \epsilon
$$

electron volts. This yields $W_{\gamma}^{D U V}=67.9 \mathrm{eV}, 61.2 \mathrm{eV}$, and $55.9 \mathrm{eV}$ for Ar, Kr, and Xe, respectively.

\section{Experimental Set-Up}

In our experimental setup (See Figure 1), we used an ${ }^{241}$ Am source which emits $\alpha$-particles of an initial energy of $5.486 \mathrm{MeV}(85 \%)$ and $5.443 \mathrm{MeV}(13 \%)$. For the measurements, $\alpha$-particles pass from an open ${ }^{241} \mathrm{Am}$ source to an APD employed as a trigger. The triggered trajectories necessarily pass through the noble gas, causing scintillation. An APD mounted on an axis perpendicular to the trajectory detects this light. The set of trajectories are contained within a cylindrical region of $1.5 \mathrm{~mm}$ radius and $45 \mathrm{~mm}$ length. The trigger is simply an APD operated in unitary gain mode.

The APDs are Advanced Photonix LAAPDs with an active diameter of $16 \mathrm{~mm}$ [8]. For scintillation light detection a windowless, DUV-enhanced device was used primarily, cross-checked against a windowless Red/IR-enhanced device.

To perform measurements, the dewar is evacuated to about $10^{-5} \mathrm{mbar}$, flushed a few times, and filled with the noble gas in which the measurement is to be performed. For the measurements in argon, the gas is purified by an Oxysorb cartridge. The condition that the $\alpha$ deposits at least $0.5 \mathrm{MeV}$ in the trigger 


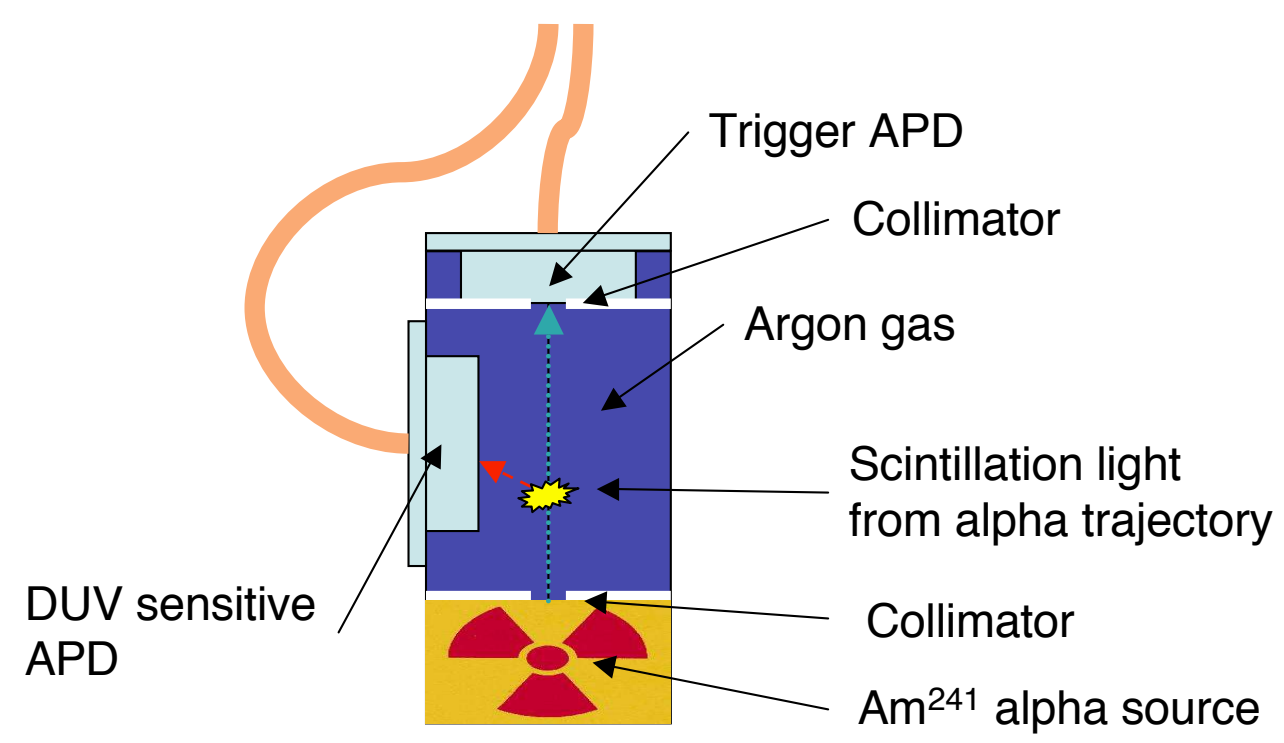

Fig. 1. Alpha particles pass from the source to an APD serving as trigger. A laterally mounted APD measures the scintillation light emitted along the trajectory.

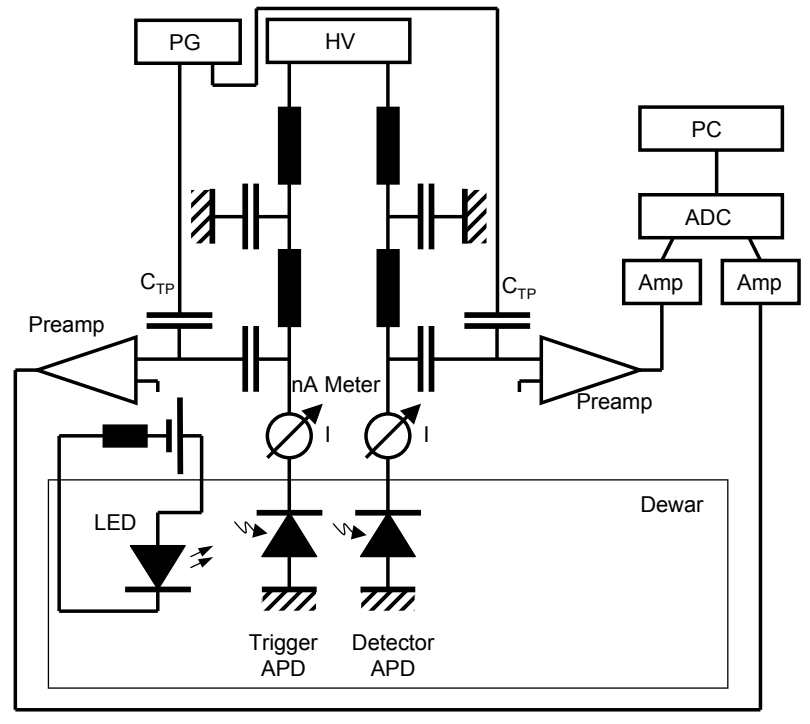

Fig. 2. Schematic of the electronics including LED and ampere meters used for calibration. Labeled components are described in text. Trigger and detector APDs have almost identical electronics chains.

imposes a gas-dependent upper limit on the pressures at which the measurements could be performed. A lower bound is given by the electrical dischargetendency encountered at low pressures. External cooling allows a gas temperature range from -5 to 20 degrees $\mathrm{C}$. Thermistors monitor the temperature of the gas as well as of the APD, the latter being of importance due to the temperature dependence of APD gain. 
A schematic of the electronics is shown in Figure 2. Directly outside the dewar, the APD signals are decoupled and amplified using a ICARUS charge-sensitive hybrid preamplifier [9]. The feedback capacitance of the preamplifier is modified to $5.7 \mathrm{pF}$ in order to be better adapted to the large capacitance of the APD. The preamplified signal is shaped and amplified by a Canberra 2020 Spectroscopy Amplifier.

The signal height is obtained by comparison with a test pulse, consisting of a pulse generator injecting a charge via a capacitor of known capacitance. Measuring the current flowing through the APD allows the gain to be monitored according to the method described in [10], where a LED situated inside the dewar serves as a continuous light source.

Typical signals are shown in Figure 3.
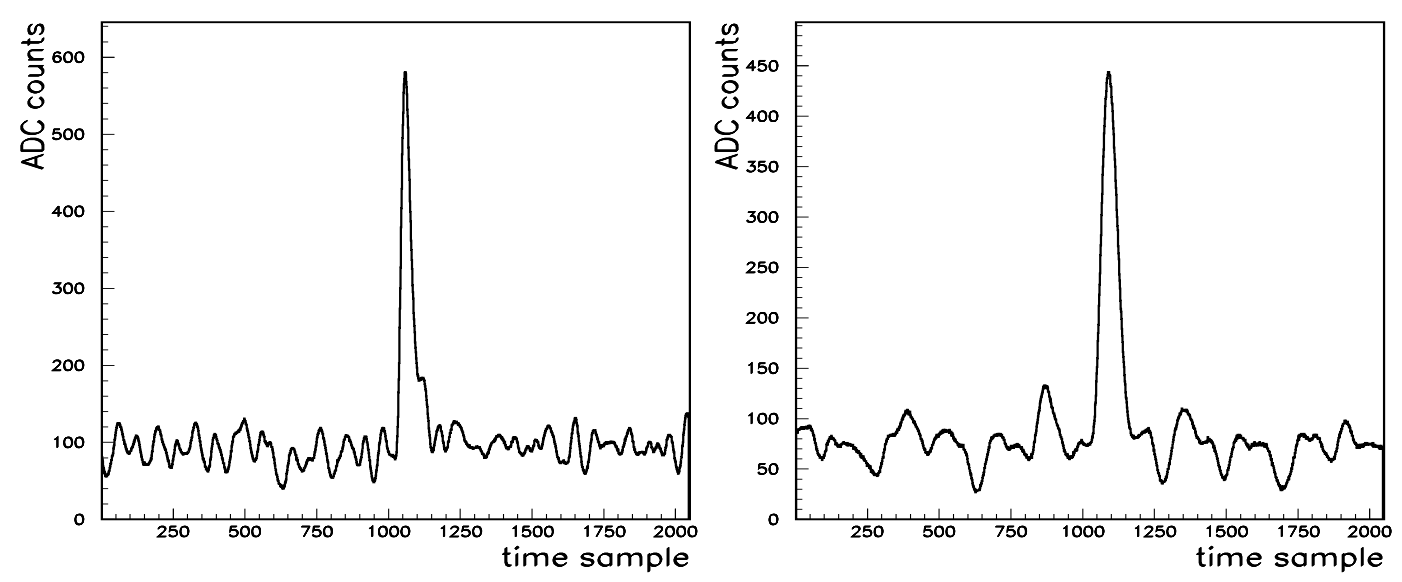

Fig. 3. Plot shows typical signals with $5 \mu$ s (left) and $10 \mu$ s shaping time (right). Time samples correspond to $400 \mathrm{~ns}$ each. The signals were randomly picked from the data taken in argon at $0.783 \mathrm{~atm}$ and $0.777 \mathrm{~atm}$ respectively, see Table 4 for more information about the conditions under which the data was acquired.

\section{Calibration}

The raw charge $Q_{S}$ of measured signals is obtained by comparison with a test pulse

$$
Q_{S}=\frac{S_{S}}{S_{T P}} \cdot Q_{T P}
$$

where $S_{S}, S_{T P}$ are the peak heights of the signal and the test pulses, respectively. The precision of the measurement depends crucially on the knowledge of the amount of charge $Q_{T P}=V_{T P} \cdot C_{T P}$ that the test pulse injects into the preamplifier. A pulse generator produces step functions of height $V_{T P}$. $V_{T P}$ is on the scale of several millivolts and can be determined with a precision better than $2 \%$. $C_{T P}$ is of the order of $5 \mathrm{pF}$. Its value can be measured more precisely 
by comparing the test pulse with the charge signal $Q_{S_{\alpha}}$ of the $5.486 \mathrm{MeV} \alpha$ ionization in the trigger APD under vacuum conditions. Since the energy $W_{S i}$ necessary to produce an electron-hole pair in the silicon of the APD is known to be $3.65 \pm 0.05 \mathrm{eV}[11,6]$,

$$
\frac{Q_{S_{\alpha}}}{e}=\left.\frac{E_{\alpha}}{W_{S i}}\right|_{v a c}
$$

where $e$ is the elementary charge. In this way, a value $C_{T P}=5.04 \pm 0.16 \mathrm{pF}$ was measured.

\section{Estimation of Absolute Photon Yield}

$W_{\gamma}^{D U V}$, the amount of energy necessary for the creation of a DUV scintillation photon in the given gas can be calculated from Equation 4 (See Section 2). Dividing the $\alpha$-particle's total energy loss in gas by this number gives an upper limit to the number of DUV photons. Quenching, or any unaccounted degree of freedom, leads to a reduction of the number of photons.

In order to correlate results from different gases, we have developed a simulation to estimate the absolute photon yield in our setup. When the $\alpha$-particles pass through the noble gas of known temperature and pressure, their energy loss along the well defined trajectory is given by [12]. We have cross-checked our calculations of the energy loss of the $\alpha$ particle by increasing the gas pressure to the threshold pressure above which the $\alpha$ loses all its energy before reaching the trigger. The calculation accurately predicts this value. The simulation assumes trajectories on straight lines. This is justified since the detour factor, defined as the ratio of the projected range to the actual length of the $\alpha$ trajectory calculated in the continuous-slowing-down-approximation, is 0.98 for $\alpha$ particles of energies around 5.5 MeV [12]. By requiring a minimal energy deposition of $0.5 \mathrm{MeV}$ in the trigger, this uncertainty is further reduced.

The detector's solid angle can be calculated for each point along the trajectory. For calculation, two models were used to simulate photon emission. The first assumes isotropic emission of photons at each point of the $\alpha$ 's trajectory. The second model assumes emission of photons in a plane perpendicular to the trajectory as suggested by [13]. Both gave comparable numbers, $N_{\text {iso }}$ being approximately $15 \%$ smaller than $N_{\text {perp }}$. Since the model of isotropic emission fits our xenon and krypton data better, $N_{i s o}$ was assumed to be the better model. In the following, $N_{\text {iso }}$ will just be referred to as $N_{\gamma}$.

An estimate of the non-UV light yield is more difficult. Although infrared emission has been detected in argon and xenon [14,15], quantitative data is hard to find in the literature. In the case of argon, we have estimated the 
non-UV contribution (See Section 9) and checked the effect on our estimation of the quantum efficiency.

\section{Quantum Efficiency}

The external quantum efficiency of APDs, from now on referred to as quantum efficiency, is defined as the number of primary electron-hole pairs produced per incident photon. The quantum efficiency $\epsilon_{Q}(\lambda)$ is a function of the wavelength of the incident light. In Figure 4, the quantum efficiency of the used LAAPD is given by the manufacturer [8].

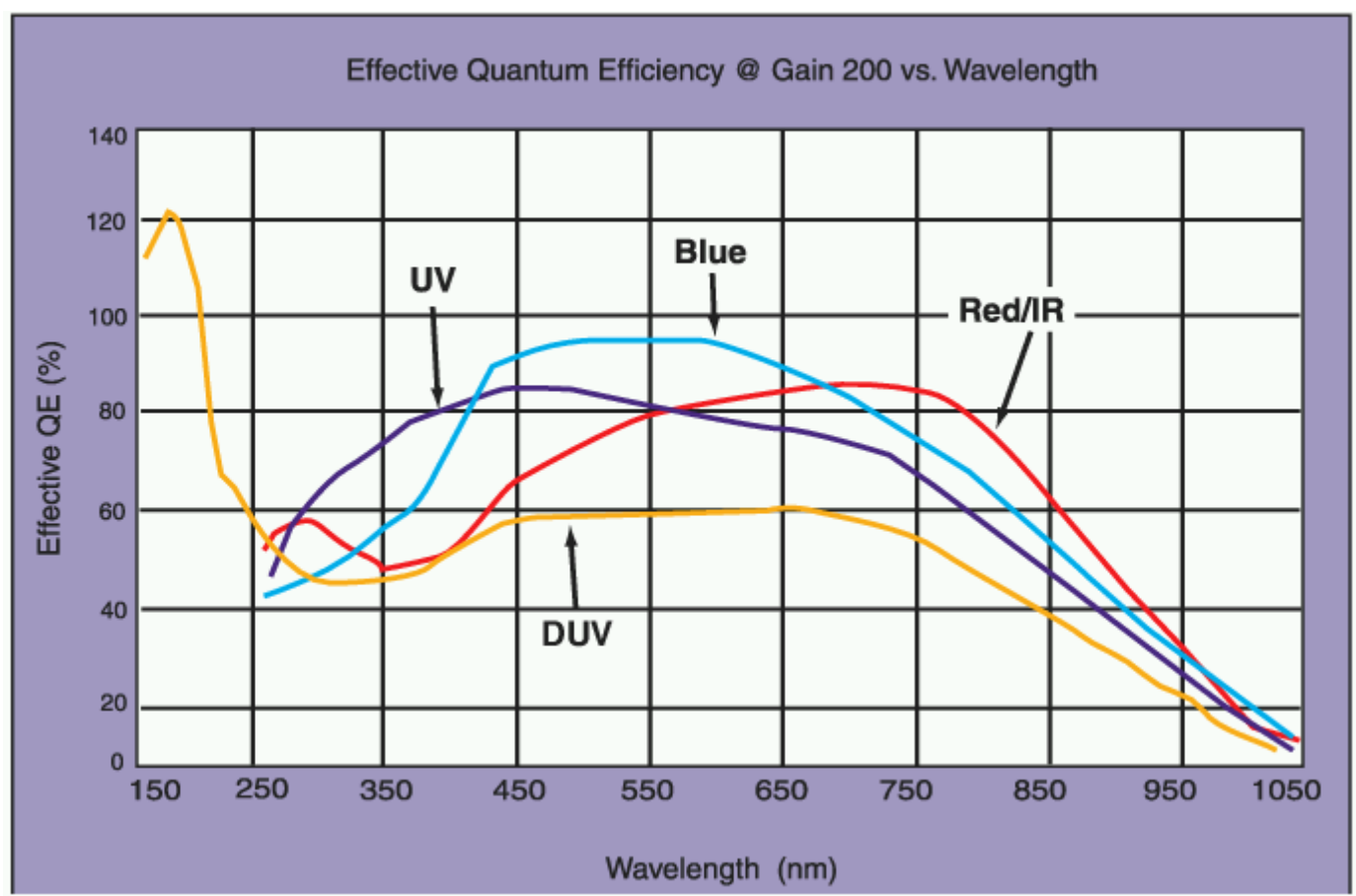

Fig. 4. The quantum efficiency of the LAAPDs [8]. The DUV enhanced device used in our measurements has a high quantum efficiency in the region of xenon scintillation light.

To our knowledge, measurements of the quantum efficiency of these devices for wavelengths below $150 \mathrm{~nm}$, specifically at the $128 \mathrm{~nm}$ of argon scintillation light, have not been published. As a reference, the highest values for the global quantum efficiency of PMTs in argon scintillation light detection were obtained by [16]. The measurements were made using a TPB wavelength shifting-coating, resulting in quantum efficiency values below $10 \%$. It is therefore quite relevant to understand the quantum efficiency of the APD also in this region of the spectrum. 


\section{Measurements}

The quantum efficiency can be obtained with

$$
\epsilon_{q 0}(128 n m)=\frac{Q_{S}}{\left(e \cdot N_{\gamma} \cdot G\right)}
$$

where $e$ is the elementary charge, $N_{\gamma}$ is the number of photons impinging on the LAAPD as predicted by our simulation (See Section 5 ), and $G$ is the measured APD gain.

Equation 7 is exact for pure argon gas with emission only at $128 \mathrm{~nm}$ detected by an idealized detector. Possible absorption effects due to impurities in the gas lead to efficiency under-estimation. Further, the employed shaping time needs to be sufficient to integrate over the full characteristic light emission time. Using an inferior shaping time leads to efficiency under-estimation. Competing with this effect is the non-UV contamination. The DUV-enhanced APD has a non-zero quantum efficiency throughout the visible and in parts of the IR region, see Figure 4. Therefore, any unaccounted non-UV contribution leads to efficiency over-estimation. In this work, the measurement of the non-UV photon contribution was performed only in argon.

To verify the correctness of our photon yield simulation, measurements were taken in krypton and xenon using DUV-enhanced LAAPD. These devices' quantum efficiencies are given by the manufacturer at the wavelengths of $\mathrm{Kr}$ and Xe DUV-emission. Tables 2 and 3 show the measured values. Errors in the last column do not include errors on $N_{\gamma}^{\text {sim }}$. The reference value of quantum efficiency given by the manufacturer is $\epsilon_{q}^{D U V}(150 \mathrm{~nm})=1.07$ and $\epsilon_{q}^{D U V}(175 \mathrm{~nm})=1.22$. Our data show good agreement with the values quoted by the manufacturer [8].

Following the same procedure, measurements were made in argon. The measured values are listed in Table 4 .

\section{Signal Length and Shaping Time}

The mechanism of DUV light emission in low pressure noble gas occurs on a time scale of several microseconds, see [13]. Therefore, not all charge is integrated when running measurements at shaping times that optimize energy resolution. Series of data (not listed in Table 4) were taken at different shaping times. The results are shown in Figure 5, where the signal height is plotted in arbitrary units as a function of the shaping time used. The data was fitted to 


\begin{tabular}{|c|c|c|c|c|c|c|}
\hline $\begin{array}{c}p_{K r} \\
(\mathrm{~atm})\end{array}$ & $\begin{array}{c}T_{K r} \\
\left({ }^{\circ} \mathrm{K}\right)\end{array}$ & $\begin{array}{c}\text { Shaping } \\
(\mu \mathrm{s})\end{array}$ & Gain & $\begin{array}{c}Q_{s} \\
(\mathrm{fC})\end{array}$ & $N_{\gamma}^{\text {sim }}$ & $\frac{Q_{S}}{e \cdot N_{\gamma}^{\text {sim }} \cdot G}$ \\
\hline \hline 0.629 & 285.0 & 10 & $26.1 \pm 1.8$ & $15.6 \pm 0.6$ & 3844 & $0.97 \pm 0.08$ \\
0.600 & 284.4 & 10 & $28.1 \pm 2.1$ & $15.8 \pm 0.6$ & 3613 & $0.97 \pm 0.08$ \\
0.573 & 284.0 & 10 & $25.1 \pm 1.6$ & $15.5 \pm 0.6$ & 3402 & $1.13 \pm 0.08$ \\
0.551 & 283.9 & 10 & $25.3 \pm 1.6$ & $14.3 \pm 0.5$ & 3246 & $1.08 \pm 0.08$ \\
0.514 & 283.9 & 10 & $27.3 \pm 1.9$ & $13.8 \pm 0.5$ & 2960 & $1.06 \pm 0.08$ \\
\hline $\mathrm{Kr}$ & DUV-enh & & & & & $1.04 \pm 0.08$ \\
\hline
\end{tabular}

Table 2

The gain-independent signal normalized to the expected number of DUV photons in krypton (See text).

\begin{tabular}{|c|c|c|c|c|c|c|}
\hline $\begin{array}{c}p_{X e} \\
(\mathrm{~atm})\end{array}$ & $\begin{array}{c}T_{X e} \\
\left({ }^{\circ} \mathrm{K}\right)\end{array}$ & $\begin{array}{c}\text { Shaping } \\
(\mu \mathrm{s})\end{array}$ & Gain & $\begin{array}{c}Q_{s} \\
(\mathrm{fC})\end{array}$ & $N_{\gamma}^{\text {sim }}$ & $\frac{Q_{S}}{e \cdot N_{\gamma}^{\text {sim }} \cdot G}$ \\
\hline \hline 0.430 & 286.4 & 10 & $26.1 \pm 1.8$ & $20.6 \pm 0.8$ & 3786 & $1.29 \pm 0.10$ \\
0.407 & 285.3 & 10 & $25.6 \pm 1.7$ & $19.1 \pm 0.7$ & 3551 & $1.31 \pm 0.10$ \\
0.379 & 284.9 & 10 & $25.6 \pm 1.7$ & $18.0 \pm 0.7$ & 3256 & $1.35 \pm 0.10$ \\
0.372 & 286.0 & 10 & $24.6 \pm 1.6$ & $16.0 \pm 0.6$ & 3163 & $1.28 \pm 0.10$ \\
\hline $\mathrm{Xe}$ & DUV-enh & & & & & $1.3 \pm 0.1$ \\
\hline
\end{tabular}

Table 3

The gain-independent signal normalized to the expected number of DUV photons in xenon (See text).

a function

$$
S(\tau)=p_{0}-p_{1} \cdot \exp \left(-p_{2} \cdot \tau\right)
$$

where $\tau$ is the shaping time in microseconds. The fitted decay frequency of $p_{2}=0.395 \mu s^{-1}$ is comparable to the value $p_{2}=0.2+0.12 \cdot p=0.297 \mu s^{-1}$ obtained by [13]. We conclude for argon pressures above .678 atmospheres, the use of $10 \mu$ s shaping time guarantees the integration of at least $94 \%$ of the full signal.

This fact is confirmed by the data taken. The series taken at $5 \mu$ s shaping time shows a clear pressure dependence, see Table 4 and Figure 6 . The series taken with $10 \mu$ s shaping time shows no systematic pressure dependence.

The energy resolution is optimized at a finite value of shaping time. For measurements performed at an APD temperature of $10^{\circ} \mathrm{C}$, the best energy resolution of $\sigma / \bar{x}=0.085$ is achieved with $2 \mu$ s shaping. As the APD temperature 


\begin{tabular}{|c|c|c|c|c|c|c|}
\hline $\begin{array}{c}p_{A r} \\
(\mathrm{~atm})\end{array}$ & $\begin{array}{c}T_{A r} \\
\left({ }^{\circ} \mathrm{K}\right)\end{array}$ & $\begin{array}{c}\text { Shaping } \\
(\mu \mathrm{s})\end{array}$ & Gain & $\begin{array}{c}Q_{s} \\
(\mathrm{fC})\end{array}$ & $N_{\gamma}^{\text {sim }}$ & $\frac{Q_{S}}{e \cdot N_{\gamma}^{\text {sim }} \cdot G}$ \\
\hline 0.835 & 278.7 & 5 & $87 \pm 6$ & $32.7 \pm 1.2$ & 3337 & $0.70 \pm 0.05$ \\
0.808 & 278.4 & 5 & $96 \pm 8$ & $32.9 \pm 1.2$ & 3181 & $0.67 \pm 0.06$ \\
0.783 & 278.4 & 5 & $63 \pm 5$ & $18.5 \pm 0.7$ & 3037 & $0.60 \pm 0.05$ \\
0.757 & 278.4 & 5 & $52 \pm 4$ & $14.5 \pm 0.5$ & 2895 & $0.60 \pm 0.05$ \\
0.727 & 278.2 & 5 & $38 \pm 3$ & $9.5 \pm 0.4$ & 2738 & $0.56 \pm 0.04$ \\
0.699 & 278.2 & 5 & $39 \pm 3$ & $8.5 \pm 0.3$ & 2598 & $0.53 \pm 0.04$ \\
\hline \hline 0.830 & 282.5 & 10 & $69 \pm 5$ & $25.6 \pm 1.0$ & 3238 & $0.72 \pm 0.05$ \\
0.777 & 281.5 & 10 & $67 \pm 5$ & $24.5 \pm 0.9$ & 2956 & $0.77 \pm 0.06$ \\
0.750 & 281.4 & 10 & $57 \pm 4$ & $17.7 \pm 0.7$ & 2815 & $0.69 \pm 0.05$ \\
0.719 & 281.3 & 10 & $57 \pm 4$ & $16.7 \pm 0.5$ & 2659 & $0.69 \pm 0.05$ \\
0.678 & 281.2 & 10 & $53 \pm 4$ & $15.5 \pm 0.7$ & 2461 & $0.74 \pm 0.06$ \\
\hline Ar & DUV-enh & & & & & $0.72 \pm 0.06$ \\
\hline
\end{tabular}

Table 4

The gain-independent signal normalized to the expected number of DUV photons in argon (See text). Measurements performed using DUV-enhanced LAAPD. The last line gives the average value of the $10 \mu$ s measurements.

\begin{tabular}{|c|c|c|c|c|c|c|}
\hline $\begin{array}{c}p_{A r} \\
(\mathrm{~atm})\end{array}$ & $\begin{array}{c}T_{A r} \\
\left({ }^{\circ} \mathrm{K}\right)\end{array}$ & $\begin{array}{c}\text { Shaping } \\
(\mu \mathrm{s})\end{array}$ & Gain & $\begin{array}{c}Q_{s} \\
(\mathrm{fC})\end{array}$ & $N_{\gamma}^{\text {sim }}$ & $\frac{Q_{S}}{e \cdot N_{\gamma}^{\text {sim.G }}}$ \\
\hline 0.835 & 275.9 & 1 & 79.9 & 10.2 & 3388 & 0.23 \\
0.820 & 276.7 & 1 & 77.6 & 9.39 & 3281 & 0.23 \\
0.806 & 276.7 & 1 & 80.0 & 9.98 & 3197 & 0.24 \\
\hline $\mathrm{Ar}$ & Red/IR-enh & & & & & 0.233 \\
\hline 0.862 & 278.7 & 1 & 90.4 & 7.2 & 3505 & 0.14 \\
0.847 & 279.2 & 1 & 159.9 & 13.6 & 3400 & 0.16 \\
0.830 & 279.1 & 1 & 168.5 & 14.5 & 3303 & 0.16 \\
\hline $\mathrm{Ar}$ & Red/IR+Filter & & & & & 0.153 \\
\hline
\end{tabular}

Table 5

Measurements performed in argon with the red/IR-enhanced LAAPD. The lower data is taken with a UV absorbing foil in front (See text). 


\section{Charge Integration}

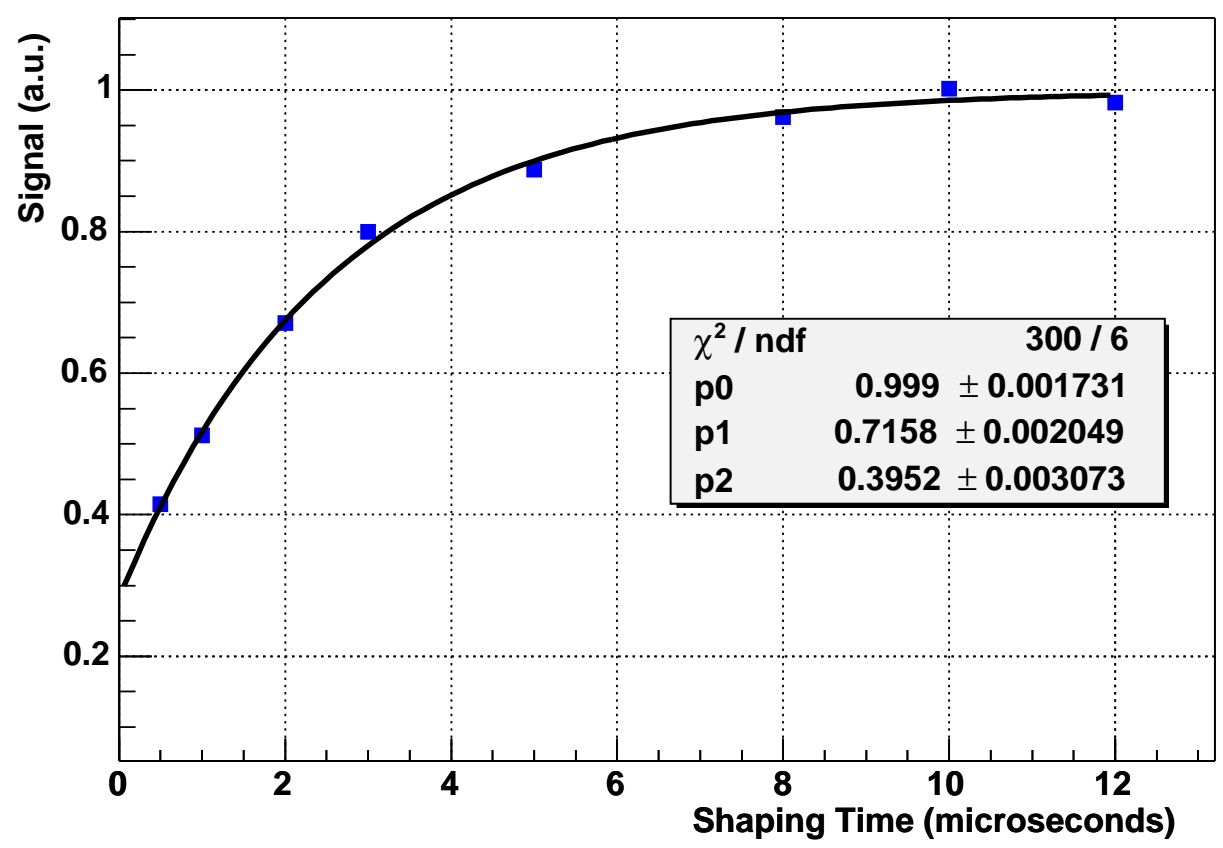

Fig. 5. Long shaping times are necessary to integrate full charge. Plot shows relative signal size for different shaping times. Data taken in argon at $0.8 \mathrm{~atm}$ and 280 degrees K. Plotted errors are solely statistical.

is reduced, dark current and consequently parallel noise decreases, reducing the loss of resolution at long shaping times.

\section{Non-UV Contribution}

Non-UV emission is assumed to be emitted from atomic transitions before dimer formation.

For argon, measurements were performed to allow the subtraction of the possible non-UV contribution. This was done by replacing the DUV-enhanced LAAPD by a red/IR-enhanced LAAPD from the same manufacturer. The measurements are shown in Table 5 . Note that $N_{\gamma}^{\text {sim }}$ in the table represents the number of expected DUV photons. The device, however, is not primarily sensitive to this wavelength. Therefore, the signal normalized to the expected number of DUV photons should not be interpreted directly as a quantum efficiency. Rather, this result is to be interpreted as a significant contribution of non-UV photons to argon scintillation light.

Because of its direct production mechanism, non-UV emission should occur on a faster time scale than DUV emission. Our non-UV measurements were 


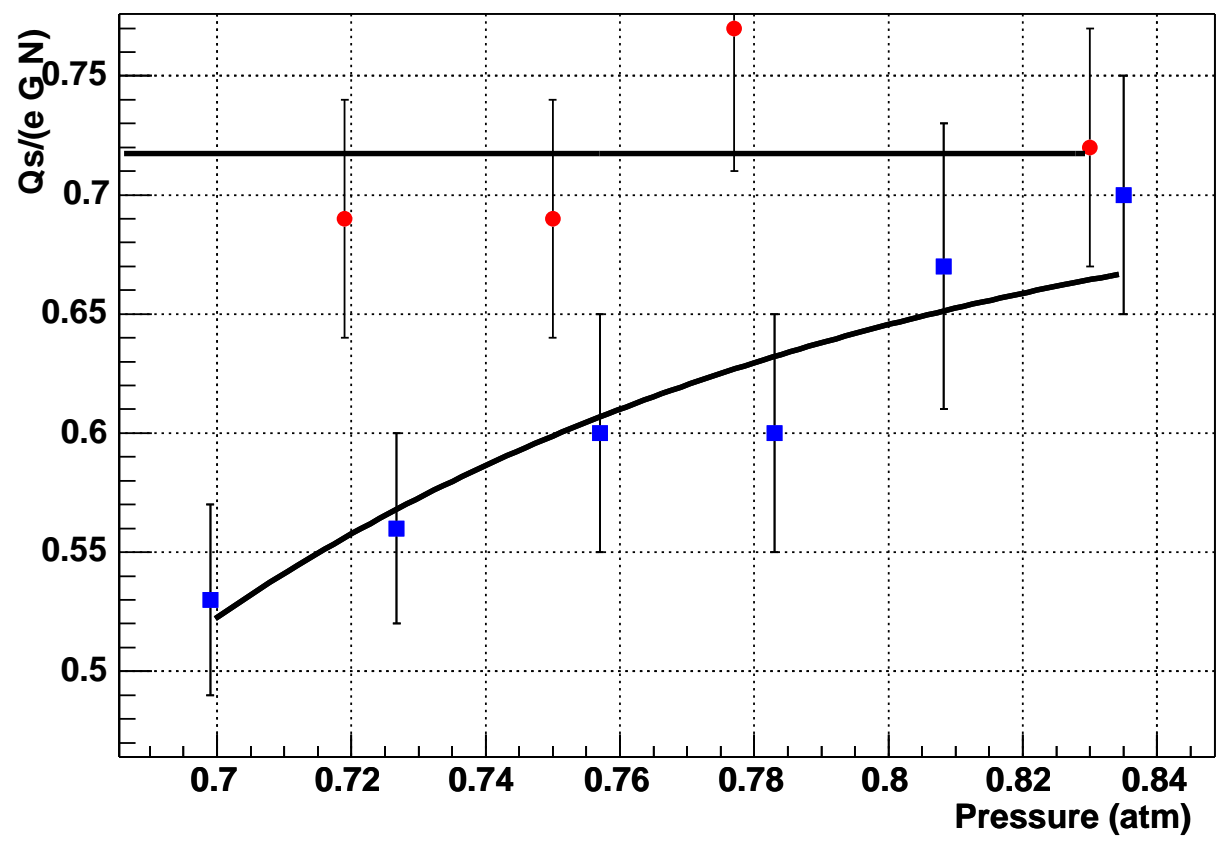

Fig. 6. The plot shows a systematic pressure dependence of data taken at short shaping times, $5 \mu \mathrm{s}$ in the case of the data plotted by the square markers. The data taken at $10 \mu \mathrm{s}$ shaping time, plotted as circles, shows no systematic pressure dependence. The error bars do not include errors on $N_{\gamma}$.

performed at $1 \mu$ s shaping time. Note from Table 5 that no systematic pressure dependence of the non-UV signal seems to be present. This observation is consistent with the attribution of non-UV emission to transitions between excited atomic states which are precursors of dimer (DUV) emission.

The red/IR enhanced LAAPD has an $\mathrm{SiO}_{2}$ anti-reflective coating. In general, $\mathrm{SiO}_{2}$ is opaque to UV light. It is not clear to what extent such an effect attenuates $128 \mathrm{~nm}$ light passing through this $150 \mathrm{~nm}$ thin coating. We do not a priori exclude a residual sensitivity of the red/IR enhanced device to DUV light.

To reduce this ambiguity, a plastic foil of $0.1 \mathrm{~mm}$ thickness was employed, mounted in front of the red/IR-enhanced device. It can be assumed that this foil has no transmittance in the DUV range, and a finite transmittance in the Red/IR range. The foil caused a signal reduction of $34 \%$, see Table 5 . This leads us to conclude that at least $65 \%$ of the unfiltered signal detected with the red/IR-enhanced LAAPD can be attributed to non-UV photons.

From now on, we use the super-index $I R$ when referring to the red/IRenhanced APD. Likewise, the super-index $D U V$ denotes the DUV-enhanced device. The number of non-UV photons is written as $N^{I R}$. Values of quantum 
efficiency always are a function of the wavelength given in parenthesis.

Using the data listed in Table 5, we can give a strict lower limit for the branching ratio $N^{I R} / N_{\gamma}^{s i m}$ of the emission of non-UV photons in argon. The number of non-UV photons impinging on the Red/IR-enhanced LAAPD relates to the detected charge signal in linear dependence of gain and quantum efficiency:

$$
N^{I R}=\frac{Q_{S}}{e \cdot \epsilon_{q}^{I R}(\lambda) \cdot G}
$$

The expression is minimized by the maximum quantum efficiency of the Red/IRenhanced APD, giving the expression

$$
\left(\frac{Q_{S}}{e \cdot N_{\gamma}^{s i m} \cdot G}\right)_{\text {Red/IR+Filter }} \cdot \frac{1}{\max _{270 \leq \lambda \leq 1050} \epsilon_{q}^{I R}(\lambda)}=0.18 \leq \frac{N^{I R}}{N_{\gamma}^{s i m}}
$$

where $\max _{\lambda}\left(\epsilon_{q}^{I R}\right)=0.85$ was used. By comparison with $W_{\gamma}^{D U V}$ (See Section 2), the obtained value can be translated into

$$
W_{\gamma}^{I R} \leq 378 \mathrm{eV}
$$

a strict upper limit for the average amount of energy needed to produce a non-UV photon in argon gas around this pressure.

With the data in Table 5, the quantum efficiency of the DUV-enhanced LAAPD for radiation at $128 \mathrm{~nm}$ can be calculated more precisely

$$
\epsilon_{q}^{D U V}(128 n m)=\epsilon_{q 0}^{D U V}(128 n m)-N^{I R}(\lambda) \cdot \epsilon_{q}^{D U V}(\lambda)
$$

where $\epsilon_{q 0}^{D U V}(128 \mathrm{~nm})$ refers to the left side of Equation 7 and the last term is the correction for the sensitivity of the DUV-enhanced device to any non-UV photons of wavelength $\lambda$ emitted.

As a strict lower limit,

$$
\left(\frac{Q_{S}}{e \cdot N_{\gamma}^{s i m} \cdot G}\right)_{D U V}-\left(\frac{Q_{S}}{e \cdot N_{\gamma}^{s i m} \cdot G}\right)_{I R} \cdot \max _{270 \leq \lambda \leq 1050} \frac{\epsilon_{q}^{D U V}(\lambda)}{\epsilon_{q}^{I R}(\lambda)} \leq \epsilon_{q}^{D U V}(128 n m),
$$

and, as an upper limit

$$
\left(\frac{Q_{S}}{e \cdot N_{\gamma}^{s i m} \cdot G}\right)_{D U V}-\left(\frac{Q_{S}}{e \cdot N_{\gamma}^{s i m} \cdot G}\right)_{I R+F i l t e r} \cdot \min _{270 \leq \lambda \leq 1050} \frac{\epsilon_{q}^{D U V}(\lambda)}{\epsilon_{q}^{I R}(\lambda)} \geq \epsilon_{q}^{D U V}(128 n m) .
$$

can be given. Thus,

$$
0.42 \leq \epsilon_{q}^{D U V}(128 n m) \leq 0.73
$$




\section{Quantum Efficiency}

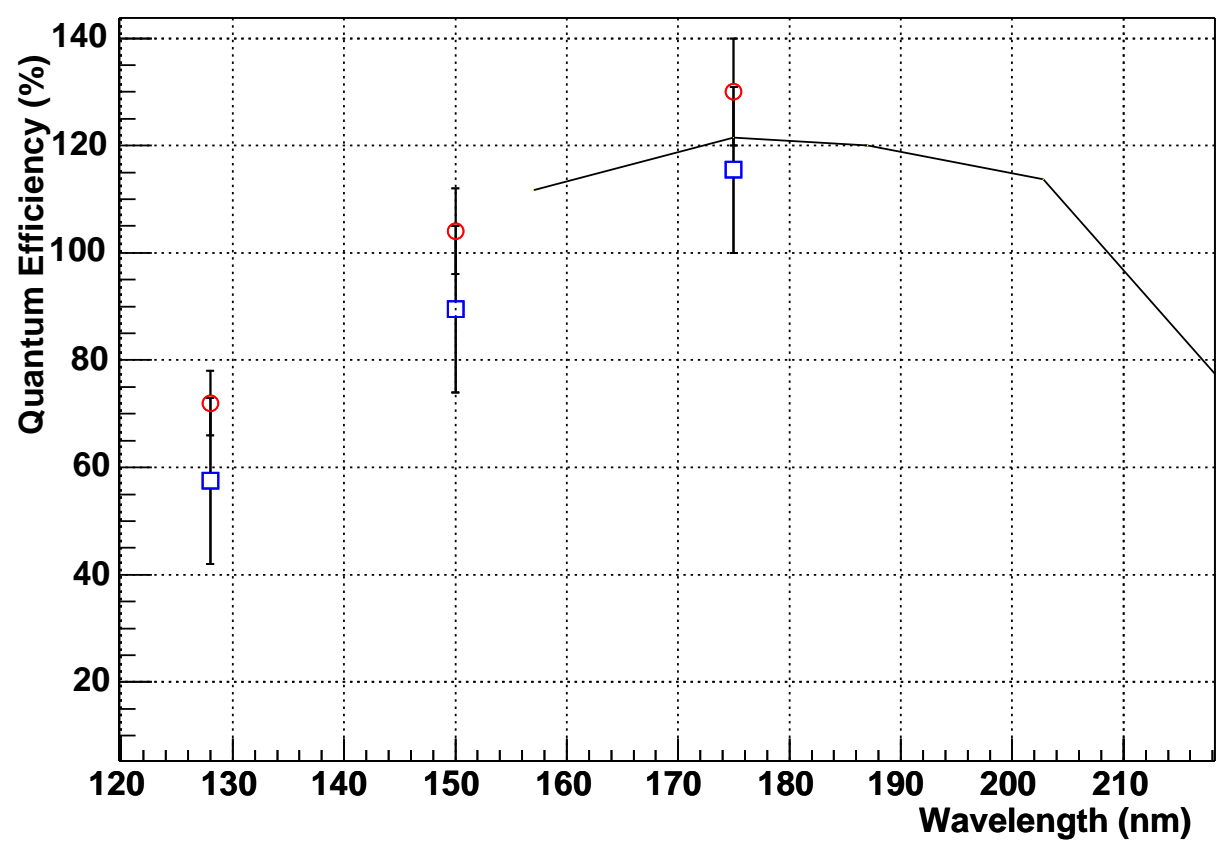

Fig. 7. The quantum efficiency of the DUV-enhanced LAAPD: The continuous curve depicts the values given by the manufacturer, see Figure 4 . The circles represent the values from Tables 4, 2, and 3 at argon, krypton, and xenon emission wavelengths. The square markers include the correction for non-UV components (see Section 9).

where the upper limit is not rigorous as quenching effects due to gas impurities have not been accounted for.

If argon non-UV emission is centered around $940 \mathrm{~nm}$ as measured by [14], this would result in an in-between value of $\epsilon_{q}^{D U V}(128 \mathrm{~nm}) \approx 0.58$. Note that over a large region of the IR spectrum, the ratio of $\epsilon_{q}^{D U V} / \epsilon_{q}^{I R}$ is relatively constant, making $\epsilon_{q}^{D U V}(128 \mathrm{~nm})$ relatively insensitive to the exact wavelength of peak IR emission.

In xenon and krypton, the non-UV contribution was not quantitatively measured.

\section{Conclusion}

Deep ultraviolet light can be detected by LAAPDs with significantly higher quantum efficiency than by conventional means such as photomultipliers.

Our data is consistent with the fact that argon gas at low pressure emits a significant amount of non-UV light. A strict upper limit for the average energy 
necessary to produce a non-UV photon in argon gas at a pressure below 1 atm is given. A lower limit cannot be given since our device is not sensitive throughout the IR full spectrum.

The given limits for the $\epsilon_{q}^{D U V}(128 \mathrm{~nm})$ apply for argon non-UV emission down to $270 \mathrm{~nm}$. Since impurity quenching was not considered, the lower limit of $42 \%$ is strict while the upper limit is not. For non-UV emission centered around $940 \mathrm{~nm} \epsilon_{q}^{D U V}(128 \mathrm{~nm}) \approx 58 \%$ is obtained.

Figure 7 summarizes the obtained results for argon, krypton, and xenon. Our measurements are consistent with the manufacturer's data where it is available. The non-UV correction was only measured for argon. For xenon and krypton this correction is of illustrative nature only. Error bars do not include inaccuracies of $N_{\gamma}^{\text {sim }}$, nor do they include quenching effects which lead to efficiency under-estimation. Our results tend to underestimate the quantum efficiency. This may be attributed to the presence of UV attenuating impurities, whose presence are not considered in the calculation. If this is the case, the effect can be expected to be more severe at shorter wavelengths. In this sense, our measurement of the quantum efficiency at $128 \mathrm{~nm}$ is to be seen as a lower limit.

\section{Acknowledgments}

We are indebted to the INFN Padova group who has cordially lent us the readout electronics necessary for the measurements. In particular, we thank Sandro Centro (INFN Padova) for his support. We acknowledge Francesco Pietropaolo and Pio Picchi who indirectly contributed to this study through useful discussions.

This work was supported by ETH/Zürich and Swiss National Science Foundation.

\section{References}

[1] T. Doke, K. Masuda, and E. Shibamura, Estimation of Absolute Photon Yields in Liquid Argon and Xenon for Relativistic (1 MeV) Electrons, Nucl. Instr. and Methods in Phys. Res. A291 (1989) 617-620.

[2] M. Suzuki, Recombination Luminescence from Ionization Tracks Produced by Alpha Particles in High pressure Argon, Krypton and Xenon Gases, Nucl. Instr. and Methods 215 (1983) 345-356. 
[3] G. S. Hurst, Vacuum Ultraviolet Radiation and Jesse Effects in the Noble Gases, Phys. Rev. A 2,5 (1970) 1717.

[4] M. Miyajima, T. Takahashi, S. Konno, T. Hamada, S. Kubota, H. Shibamura, and T. Doke, Average energy expended per ion pair in liquid argon, Phys. Rev. A $9,3(1974) 1438$.

[5] R. L. Platzman, Total Ionization in Gases by High-Energy Particles: An Appraisal of Our Understanding, Int. J. Appl. Radiat. and Isot. 10 (1961) 116.

[6] International Commission on Radiation Units and Measurements, Average energy required to produce an ion pair, 1979.

[7] M.J. Carvalho and G. Klein, Alpha-Particle Induced Scintillation in Dense Gaseous Argon: Emission Spectra and Temporal Behaviour of its Ionic Component, Nucl. Instr. and Methods 178 (1980) 469-475.

[8] Advanced Photonix, Inc. See http://www.advancedphotonix.com/

[9] S. Amerio, M. Antonello, B. Baiboussinov, S. Centro, F. Pietropaolo, W. Polchlopek, S. Ventura, Considerations on the ICARUS read-out and on data compression, Internal Note ICARUS-TM/2002-05.

[10] A. Karar, Y. Musienko, and J. Ch. Vanel, Characterization of avalanche photodiodes for calorimetry applications Nucl. Instr. and Methods in Phys. Res. A 428 (1999) 413-431.

[11] E. Aprile, A. Bolotnikov, D. Chen, and R. Mukherjee, $W$ value in liquid Krypton, Phys. Rev. A 48, 2 (1993) 1313.

[12] M.J. Berger, J.S. Coursey, and M.A. Zucker Stopping-Power and Range Tables for Electrons, Protons, and Helium Ions, http://physics.nist.gov/PhysRefData/Star/Text/contents.html

[13] P. Moerman, R. Boucique, and P. Mortier, Pressure Dependendent Decay of the Ultraviolet Continuum of Argon Phys. Lett. A 49, 2 (1974) 179.

[14] G. Bressi, G. Carugno, E. Conti, D. Iannuzzi, A.T. Meneguzzo, A first study of the infrared emission in argon excited by ionizing particles, Phys. Lett. A 278 (2001) 280-285.

[15] S.Belogurov, G. Bressi, G. Carugno, E. Conti, D. Iannuzzi, A. T. Meneguzzo, Measurement of the light yield of infrared scintillation in xenon gas, Nucl. Instr. and Methods in Phys. Res. A 452(2000) 167-169.

[16] P. Benetti, C. Montanari, G. L. Raselli, M. Rossella, and C. Vignoli Detection of the VUV liquid argon scintillation light by means of glass-window photomultiplier tubes, Nucl. Instr. and Methods in Phys. Res. A 505 (2003) 89-92. 